\title{
Alveolar soft part sarcoma presenting as asymptomatic pulmonary nodules:- report of a case with ultrastructural diagnosis
}

\author{
JF CORDIER, C BAILLY, E TABONE, F CHEIX, J BRUNE, R TOURAINE \\ From the Centre Hospitalier Universitaire and Centre Léon Bérard, Lyon, France
}

Lung metastases are frequently the mode of presentation of alveolar soft part sarcoma,' a rare tumour of the skeletal muscles of the thigh and arm occurring between the ages of about 20 and 30 years, with a female predominance. ${ }^{2}$ Although the pathological features of this tumour are fairly characteristic, it is the demonstration by electron microscopy of typical intracellular crystals that is pathognomonic. ${ }^{34}$

We report a case of alveolar soft part sarcoma presenting as asymptomatic pulmonary nodules, without evidence of a primary tumour, in which the diagnosis was established by electron microscopy of material obtained by lung biopsy.

\section{Case report}

A symptomless 20 year old white man was referred to us in October 1981 for diagnosis of multiple pulmonary nodules seen on a routine chest radiograph. He was a non-smoker and had no previous history of illness. A chest radiograph obtained two years previously was normal. Physical examination revealed no abnormality. The chest radiograph showed multiple small ( $<15 \mathrm{~mm}$ ) bilateral nodules, mainly in the lung bases. The findings at fibreoptic bronchoscopy were normal. Results of routine haematological, bacteriological, and biochemical studies were unremarkable. Extensive investigation for a non-pulmonary primary malignant tumour was negative. Tissue obtained from an open lung biopsy of a nodule in the left lower lobe was examined by light microscopy and a provisional diagnosis of either a chemodectoma or an alveolar soft part sarcoma was made. From December 1981 to May 1982 seven courses of chemotherapy (doxorubicin, cyclophosphamide, vincristine, dacarbazine) were given, one every 28 days. There was no change in the chest radiographic appearances and treatment was discontinued.

In July 1983 the patient was readmitted to hospital because of increase in size of a single lingular nodule. The lingula was resected surgically and found to contain a firm white nodule $2 \mathrm{~cm}$ in diameter, one nodule $3 \mathrm{~mm}$ in diameter, and 12 nodules less than $1 \mathrm{~mm}$ in diameter.

Tumour tissues were routinely processed for light microscopy and small fragments of tumour were immediately

Address for reprint requests: JF Cordier, Hôpital Louis Pradel, BP
Lyon Montchat, 69394 Lyon, Cedex 03, France.

Accepted 5 October 1984

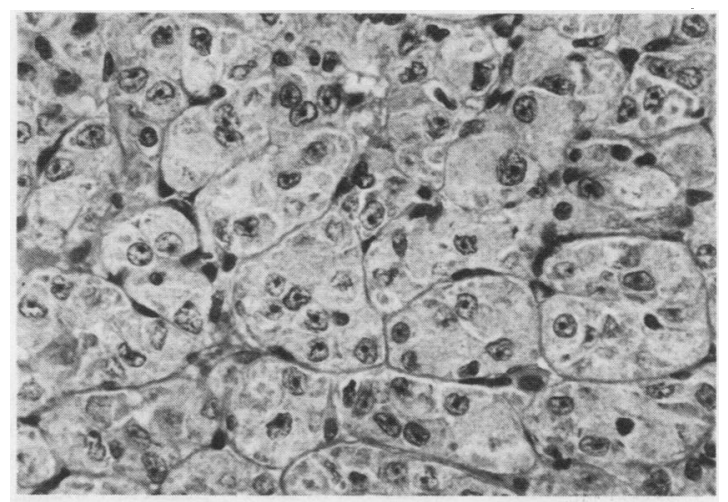

Fig 1 Light microscopic appearance of the alveolated pattern of the sarcoma, groups of round or polygonal cells circumscribed by connective tissue with capillaries.

(Hemalun-Phloxin-Safran, $\times$ 240.)

fixed in $0.1 \mathrm{mmol} / \mathrm{l}$ sodium cacodylate buffer containing $2 \%$ glutaraldehyde and processed for electron microscopy.

Microscopic examination showed well defined nodules consisting of polyhedral granular tumour cells arranged in clusters, surrounded by dense, well vascularised fibrous trabeculae (fig 1). This endocrine like alveolar pattern was accentuated by the reticulin staining. The argyrophil reaction was negative. The periodic acid Schiff (PAS) reaction was slightly positive, but no crystalline formations were seen by light microscopy. No mitosis and no atypia were noted.

Electron microscopic examination showed the tumour to be composed of solid nests of closely packed tumour cells, surrounded by a thick, well defined basal lamina, which also extended as a thin layer between individual cells. The alveoli were separated by mesenchymal cells and capillaries and often contained both dark and light cells. A few hemidesmosomes facing the perialveolar basement membrane were seen. The nuclei were clear with a smooth profile and irregular folds and had prominent nucleoli. The cytoplasmic matrix was filled with numerous swollen mitochondria and a variable number of rhomboidal or polygonal crystalline inclusions. These crystals were often close to small dense vesicles of well developed Golgi complexes (fig 2). When seen in the correct plane of section, 


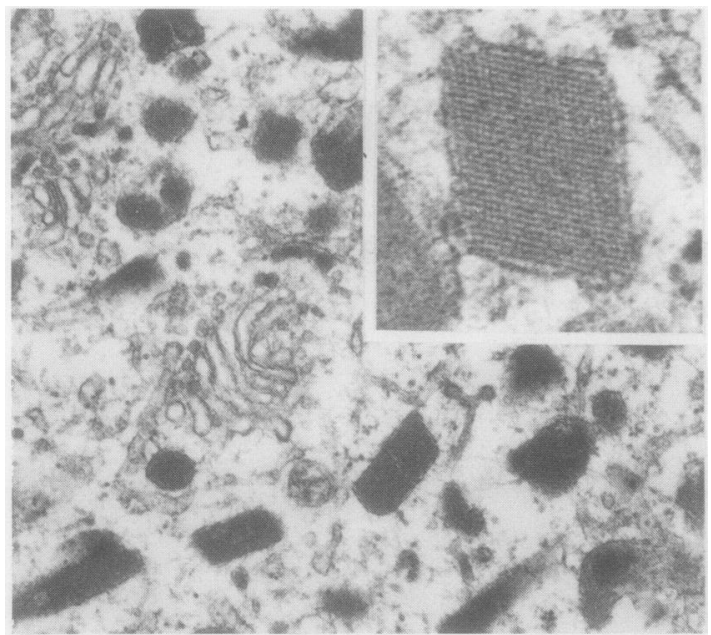

Fig 2 High power electron micrograph showing the close association of the crystalline inclusions with the well developed Golgi complex. The insert shows the periodic pattern of $10 \mathrm{~nm}$ and the surrounding membrane of a crystal. $(\times 15$ 000; insert $\times 30000$. $)$

the crystals had a periodic pattern of $10 \mathrm{~nm}$ and were surrounded by a membrane (fig 2 , insert). There was much rough endoplasmic reticulum, which in some areas of the cytoplasmic matrix was stacked in closely packed, winding, or concentric arrays.

\section{Discussion}

Pulmonary metastases are common in alveolar soft part sarcoma and are often present at the time of diagnosis. ${ }^{\prime 2}$ Lung metastases appear as either solitary or multiple nodules; their growth is usually slow, but fatal. We could find only three reported cases of alveolar soft part sarcoma presenting as pulmonary nodules: in one patient the biopsy diagnosis of a papillary adenoma was later changed to one of primary alveolar soft part sarcoma'; another patient was thought on the basis of the lung biopsy to have a metastatic hypernephroma until alveolar soft part sarcoma of thigh was diagnosed.' The third patient had no primary tumour, but no electron microscopy study was done. ${ }^{5}$ Alveolar soft part sarcoma has often been confused with many other tumours; it consists of nests of cells, separated by vascular connective tissue. The cytoplasm is abundant and eosinophilic, the nuclei are uniform, and mitotic figures are rare. The cells appear as dark or light, depending on the number of organelles. A prominent Golgi complex and dense secretory granules are present. ${ }^{34}$ Nevertheless, these features of alveolar soft part sarcoma are remarkably similar to those of a chemodectoma. ${ }^{4}$ Since multiple primary chemodectomas or metastases of extrapulmonary chemodectoma can present as pulmonary nodules ${ }^{67}$ the crystals in alveolar soft part sarcoma cells are of great diagnostic value. These crystals usually stain with PAS and are resistant to diastase digestion. On electron microscope examination they are seen to be membrane bounded and show a periodic pattern of "lines" set $10 \mathrm{~nm}$ apart, which in transverse section are seen as irregularly outlined "points."

These lines are currently considered diagnostic of alveolar soft part sarcoma and are thought to arise from the dense granules in the cells. In our patient the crystals could not be seen by light microscopy and did not stain the PAS, but their electron microscopic morphology was typical.

The origin of alveloar soft part sarcoma is still not known, and its treatment is not well established. Resection of the nodules may be beneficial and radiotherapy and chemotherapy have occasionally produced regression or control of metastases ${ }^{9}$ but no results of controlled studies are available.

We thank D Lievre for computer search of the literature, LD Gruer for reviewing the translation of the paper and MC Thevenet for secretarial assistance.

\section{References}

1 Hurt R, Bates M, Harrison W. Alveolar soft part sarcoma. Thorax 1982;37:877-86.

2 Enzinger FM, Sharon WW. Soft tissue tumours. St Louis: CV Mosby Co, 1983.

3 Ghadially FN. Diagnostic electron microscopy of tumours. London: Butterworths, 1980.

4 Unni KK, Soule EH. Alveolar soft part sarcoma. An electron microscopic study. Mayo Clin Proc 1975;50:591-8.

5 McGlamory JC, Harris JO. Alveolar soft part sarcoma. Report of a case presenting as asymptomatic pulmonary nodules. Chest 1972;62:762-3.

6 Singh G, Lee RE, Brooks DH. Primary pulmonary paraganglioma. Report of a case and review of the literature. Cancer 1977;40:2286-9.

$7 \mathrm{Tu}$ H, Bottomley RH. Malignant chemodectoma presenting as a miliary pulmonary infiltrate. Cancer 1974;33:244-9.

8 Shipkey FH, Lieberman PH, Foote FW Jr, Stewart FW. Ultrastructure of alveolar soft part sarcoma. Cancer 1964;17:821-30.

9 Baum ES, Fickenscher L, Nachman JB, Idriss F. Pulmonary resection and chemotherapy for metastatic alveolar soft part sarcoma. Cancer 1981;47:1946-8. 